\title{
Detección de antígenos de Neisseria meningitidis del grupo B con la prueba MB-Dot-ELISA en pacientes con meningitis ${ }^{1}$
}

\author{
Maria das Graças Adelino Alkmin, ${ }^{2}$ Ilka Maria Landgraf ${ }^{3}$ \\ y Sumie Hoshino Shimizu²
}

RESUMEN La infección por Neisseria meningitidis del grupo B ha sido difícil de detectar, en parte porque el polisacárido de este grupo de bacterias es un inmunógeno débil. En este artículo se describe el análisis de la validez de una nueva prueba, la MB-Dot-ELISA, en la cual se emplea un antisuero de caballo de alta titulación para detectar antígenos de N. meningitidis del grupo B. En el estudio se valoraron muestras de líquido cefalorraquídeo de 585 personas, 574 de las cuales padecían presuntamente meningitis y 11, trastornos neurológicos. La sensibilidad de la prueba fue 0,991 y la especificidad, 0,826. Estos valores fueron más altos que los de la aglutinación en látex y son muy compatibles con los de la contrainmunoelectroforesis y los métodos de análisis bacteriológico. La prueba MB-Dot-ELISA es sensible, barata y apropiada para realizar investigaciones en los laboratorios de salud pública.

Desde 1988, se han producido brotes epidémicos de meningitis cerebroespinal causados por Neisseria meningitidis del grupo B en la ciudad de São Paulo, Brasil. Datos recientes sugieren que la incidencia de la enfermedad se está reduciendo algo, ya que en 1993 se notificaron solo 9,56 casos por millón de habitantes en comparación con 14,25 en 1990 (1).

\footnotetext{
Se publicó en inglés en el Bulletin of the Pan American Health Organization, 1996, Vol. 30, No. 3, con el título "Detection of N. meningitidis Group B antigens by MB-Dot-ELISA in patients with meningitis".

2 Instituto Adolfo Lutz, Sección de Inmunología. Las solicitudes de separatas deben dirigirse a esta primera autora en el Instituto Adolfo Lutz. Dirección postal: Avda. Dr. Arnaldo 355, 01246-902, São Paulo, SP, Brasil.

3 Instituto Adolfo Lutz, Sección de Bacteriología, São Paulo, SP, Brasil.
}

La detección de antígenos de N. meningitidis del grupo B en el líquido cefalorraquídeo (LCR) de los pacientes plantea algunas dificultades. Estas derivan de la falta de un buen antisuero específico del grupo que, a su vez, se debe a la baja inmunogenicidad del polisacárido de este grupo bacteriano (2-3). En general, los datos disponibles al respecto son escasos.

Se ha descrito el uso de una prueba de inmunovaloración en mancha (dotimmunobinding assay) en la cual se utiliza un anticuerpo monoclonal para detectar antígenos polisacáridos de meningocócicos del grupo B en el LCR y la orina de pacientes infectados (2). Si bien esta prueba parece ser más sensible que la contrainmunoelectroforesis (CIE), la aglutinación en látex (LA) o la valoración por inmunosorción enzimática (ELISA), su positividad no sobrepasó $67 \%$. No se han encontrado referencias bibliográficas sobre antisueros policlonales contra $N$. meningitidis del grupo $\mathrm{B}$.

Con objeto de mejorar los instrumentos disponibles para detectar este agente causal de la meningitis, se preparó con anterioridad a este estudio un antisuero policlonal de alta titulación contra N. meningitidis del grupo B en caballos (4), que suele usarse en el diagnóstico por CIE. El trabajo que aquí se presenta se llevó a cabo con el fin de estandarizar y evaluar otra prueba, la dot-ELISA para meningococos del grupo B (MB-Dot-ELISA), y comparar los resultados obtenidos con los de la microscopia, los métodos de cultivo y otras técnicas de análisis inmunológico como la CIE y la LA. 


\section{MATERIALES Y MÉTODOS}

\section{Líquido cefalorraquídeo}

En este estudio se analizaron 585 muestras de LCR. De ellas, 574 se habían enviado al Instituto Adolfo Lutz para diagnóstico inmunobacteriológico después de obtenerlas de pacientes hospitalizados en el Instituto de Infectología Emilio Ribas por presentar presuntos casos de meningitis. Las 11 muestras de LCR restantes se extrajeron de pacientes que padecían trastornos neurológicos y estaban ingresados en la policlínica de la Facultad de Medicina de la Universidad de São Paulo.

De las 574 muestras, 113 eran de pacientes infectados por N. meningitidis del grupo B. Otras 287 fueron positivas en el examen microscópico (tinción de Gram), el cultivo $u$ otros métodos de análisis bacteriológico para detectar otros agentes etiológicos (5-6). Las 174 restantes fueron negativas. Los resultados de las 11 muestras tomadas en el Hospital Universitario de São Paulo fueron negativos. Todas las muestras de LCR se procesaron en un período de 24 a 48 horas mediante métodos bacteriológicos y $\mathrm{CIE}$, y se guardaron a temperaturas por debajo de $-20^{\circ} \mathrm{C}$ durante casi un año para utilizarlas en otras pruebas inmunológicas.

\section{Técnicas inmunológicas convencionales}

La CIE se realizó como se indicó anteriormente (7). Para la prueba de LA, se empleó un estuche comercial de reactivos (Biolab Diagnóstico SA, bioMérieux 69260 Charbonnières les Bains, Francia), que fue donado por el Dr. Antonio Walter Ferreira del Instituto de Medicina Tropical de São Paulo.

\section{MB-Dot-ELISA}

La prueba MB-Dot-ELISA se estandarizó del siguiente modo: se trazaron áreas de $0,5 \times 0,5 \mathrm{~cm}$ en tiras de nitrocelulosa de $1,0 \times 11,0 \mathrm{~cm}$ con un marcador indeleble. Luego se hizo una mancha en el centro de cada cuadrilátero con una muestra de $1,0 \mu \mathrm{L}$ de LCR. Los controles positivos fueron un antígeno polisacárido de $N$. meningitidis del grupo B (8) y una muestra de LCR de un paciente infectado por esta bacteria. Los controles negativos fueron muestras de LCR de pacientes con infecciones distintas de la meningitis. Después de secar las tiras a temperatura ambiente, se bloqueó la membrana con gelatina al 3\% en una solución salina con tampón fosfato (PBS), a un $\mathrm{pH}$ de 7,4, durante 30 minutos a temperatura ambiente y se lavó tres veces en agua destilada. A continuación, cada tira se incubó con 2,5 mL de antisuero de caballo contra N. meningitidis del grupo B (4), a una dilución de 1:600 en PBS, que contenía gelatina al $1 \%$ (PBS-G), durante 2 horas, a temperatura ambiente y en un agitador de plataforma oscilante. Después, se lavaron las bandas dos veces en PBS que contenía Tween 20 al 0,05\%, y se incubaron durante 2 horas con 2,5 mL de un conjugado de peroxidasa IgG anticaballo (Sigma Chemical Company, Estados Unidos de América) a una dilución de 1:500 en PBS-G. Tras otros dos ciclos de lavado con agua destilada y PBS, las tiras se incubaron con una solución cromógena (tetraclorhidrato de 3-3'diaminobencidina y $\mathrm{H}_{2} \mathrm{O}_{2}$ ) durante 5 minutos. Seguidamente, las tiras se lavaron tres veces en agua destilada. Se registró una reacción positiva al observar una mancha de color contra el fondo blanco del papel de filtro y una negativa, cuando no se observó ninguna mancha teñida.

La absorción de antisuero con antígenos de bacterias heterólogas se realizó según el método descrito por Alkmin et al. (4). Para evaluar la reproducibilidad interensayo, se analizaron 50 muestras de LCR con la prueba MBDot-ELISA en días distintos. Para evaluar la reproducibilidad intraensayo, se examinaron cinco veces las 10 muestras negativas y las 10 positivas de LCR.

Para evaluar la estabilidad de las membranas de nitrocelulosa manchadas, se prepararon cuatro membranas de cada lote de muestras de LCR y se mantuvieron entre papel secante. Des- pués de procesar la primera de ellas y antes de examinarlas, dos membranas se mantuvieron a $37^{\circ} \mathrm{C}$ durante 15 y 30 días, respectivamente, y la cuarta se mantuvo a temperatura ambiente y se procesó 6 meses más tarde.

\section{Análisis estadístico}

Para valorar la validez diagnóstica de la prueba MB-Dot-ELISA, se estimaron su sensibilidad, especificidad, valores predictivos positivos y negativos (con sus correspondientes intervalos de confianza de 95\%). La concordancia entre las pruebas diagnósticas se estimó mediante el estadístico kappa $(k)(9-13)$. El grado de significación estadística de $k$ se calculó mediante la prueba $z$, con un nivel de significación de 0,05.

\section{RESULTADOS}

Los resultados obtenidos con la prueba MB-Dot-ELISA se presentan en el cuadro 1. Como puede apreciarse, 112 de los 113 casos comprobados de infección por meningococos del grupo $B$ fueron positivos en la prueba MBDot-ELISA (lo cual corresponde a una sensibilidad de 0,991 ). Los porcentajes de positividad general calculados en el análisis de 39 muestras con LA y MB-Dot-ELISA (que comprendían 30 de esos 113 casos y de 9 de los causados por otros microorganismos causantes de meningitis) fueron $66,7 \%$ y $71,8 \%$, respectivamente. Los porcentajes de positividad verdadera de las dos pruebas fueron 89,3 y $100 \%$, respectivamente.

De las 98 muestras de LCR positivas en el análisis microscópico de diplococos gramnegativos, pero negativas en el cultivo y la CIE, $83(84,7 \%)$ fueron positivas en la prueba MB-Dot-ELISA (véase el cuadro 1). También hubo 33 casos $(17,8 \%)$ de reactividad cruzada con bacterias no relacionadas. En el grupo de 185 muestras de LCR negativas, $38(20,5 \%)$ fueron positivas en la prueba MB-Dot-ELISA.

Estos datos proporcionaron la base para evaluar el rendimiento diagnós- 
CUADRO 1. Resultados positivos y negativos obtenidos con la prueba MB-Dot-ELISA en 400 muestras de líquido cefalorraquídeo de pacientes infectados por Neisseria meningitidis del grupo B u otros agentes patógenos y 185 muestras de pacientes sin agentes patógenos detectados

\begin{tabular}{|c|c|c|c|}
\hline \multirow{2}{*}{$\begin{array}{l}\text { Agentes infecciosos detectados } \\
\text { por microscopia, cultivo o } \\
\text { contrainmunoelectroforesis }\end{array}$} & \multicolumn{3}{|c|}{$\begin{array}{l}\text { Resultados del análisis del } \\
\text { líquido cefalorraquídeo (LCR) } \\
\text { con la prueba MB-Dot-ELISA }\end{array}$} \\
\hline & $\begin{array}{l}\text { No. } \\
\text { total }\end{array}$ & $\begin{array}{c}\text { No. } \\
\text { positivos }\end{array}$ & $\begin{array}{c}\text { No. } \\
\text { negativos }\end{array}$ \\
\hline \multirow{2}{*}{\multicolumn{4}{|c|}{$\begin{array}{l}\text { Pruebas positivas: } \\
\text { Neisseria meningitidis } \\
\text { grupo B }\end{array}$}} \\
\hline & 113 & 112 & 1 \\
\hline Diplococos gramnegativos & 98 & 83 & 15 \\
\hline \multicolumn{4}{|l|}{$\begin{array}{l}\text { Otros microorganismos: } \\
\text { Neisseria meningitidis }\end{array}$} \\
\hline $\begin{array}{l}\text { grupo A } \\
\text { Neisseria meningitidis }\end{array}$ & 4 & 1 & 3 \\
\hline $\begin{array}{l}\text { grupo C } \\
\text { Neisseria meningitidis }\end{array}$ & 11 & 3 & 8 \\
\hline $\begin{array}{l}\text { grupo W135 } \\
\text { Neisseria meningitidis }\end{array}$ & 11 & 5 & 6 \\
\hline $\begin{array}{l}\text { grupo Y } \\
\text { Haemophilus influenzae }\end{array}$ & 2 & 2 & 0 \\
\hline tipo b & 80 & 8 & 72 \\
\hline Streptococcus pneumoniae & 16 & 3 & 13 \\
\hline Streptococcus sp. & 4 & 1 & 3 \\
\hline Staphylococcus aureus & 13 & 2 & 11 \\
\hline Escherichia coli & 1 & 0 & 1 \\
\hline Klebsiella sp. & 1 & 0 & 1 \\
\hline Proteus sp. & 2 & 1 & 1 \\
\hline Listeria monocytogenes & 2 & 0 & 2 \\
\hline Diplococos grampositivos & 21 & 3 & 18 \\
\hline Cocos grampositivos & 3 & 1 & 2 \\
\hline Bacilos gramnegativos & 12 & 2 & 10 \\
\hline Bacilos grampositivos & 2 & 1 & 1 \\
\hline Levadura & 4 & 0 & 4 \\
\hline Subtotal de positivas & 400 & 228 & 172 \\
\hline Pruebas negativas: & & & \\
\hline Subtotal de negativas & 185 & 38 & 147 \\
\hline Total & 585 & 266 & 319 \\
\hline
\end{tabular}

tico de la prueba MB-Dot-ELISA y otras pruebas. Como indica el cuadro 2 , el rendimiento diagnóstico de la prueba MB-Dot-ELISA no puede distinguirse del de la microscopia, el cultivo y la CIE, ya que los valores de los intervalos de confianza de $95 \%$ correspondientes a cada técnica se solapan.

En el cuadro 2 se comparan la prueba MB-Dot-ELISA con otras tres pruebas. Puesto que la sensibilidad de esas pruebas suele ser baja, la MB-DotELISA se comparó luego con una combinación de las tres, utilizando los siguientes criterios: todas las muestras de LCR positivas en el cultivo solo o en combinación con una de las otras dos pruebas o con ambas se consideraron positivas verdaderas. Además, todas las muestras positivas en la microscopia y la CIE se consideraron positivas verdaderas. Sin embargo, todas las muestras que solo fueron positivas en la microscopia o solo en la CIE se consideraron negativas. Al aplicar estos criterios, la sensibilidad y especificidad de la prueba MB-Dot-ELISA mejoraron, y sus valores ascendieron a 0,991 y 0,826, respectivamente.

La concordancia entre la prueba MB-Dot-ELISA y otras técnicas de diagnóstico, individualmente o en combinación, se estimó mediante el índice kappa $(k)$ (cuadro 3). Los valores de $k$ fueron estadísticamente significativos en todas las comparaciones, ya que los valores $z$ oscilaron entre

CUADRO 2. Rendimiento diagnóstico de la prueba MB-Dot-ELISA en comparación con las técnicas de microscopia, cultivo y contrainmunoelectroforesis (13) en el estudio de 585 muestras de líquido cefalorraquídeo. Sensibilidad = muestras positivas verdaderas detectadas/ (positivas verdaderas + negativas falsas); especificidad = negativas verdaderas detectadas/(negativas verdaderas + positivas falsas); eficiencia $=$ (positivas verdaderas detectadas + negativas verdaderas detectadas $) /$ total de muestras analizadas; valor predictivo positivo $=$ positivas verdaderas detectadas/(positivas verdaderas + positivas falsas); y valor predictivo negativo = negativas verdaderas detectadas/(negativas verdaderas + negativas falsas). En cada caso, se presenta el intervalo de confianza de $95 \%$ entre paréntesis (IC95\%)

\begin{tabular}{|c|c|c|c|c|c|c|}
\hline \multirow[b]{2}{*}{ Técnica } & \multicolumn{6}{|c|}{ Prueba MB-Dot-ELISA } \\
\hline & Positividad \% & Sensibilidad & Especificidad & Eficiencia & $\begin{array}{c}\text { Valor } \\
\text { predictivo } \\
\text { negativo }\end{array}$ & $\begin{array}{c}\text { Valor } \\
\text { predictivo } \\
\text { positivo }\end{array}$ \\
\hline Microscopia & 19,5 & $\begin{array}{c}0,987 \\
(0,930-0,998)\end{array}$ & $\begin{array}{c}0,726 \\
(0,675-0,773)\end{array}$ & $\begin{array}{c}0,777 \\
(0,734-0,815)\end{array}$ & $\begin{array}{c}0,996 \\
(0,976-0,999)\end{array}$ & $\begin{array}{c}0,466 \\
(0,391-0,543)\end{array}$ \\
\hline Cultivo & 14,9 & $\begin{array}{c}0,983 \\
(0,910-0,997)\end{array}$ & $\begin{array}{c}0,699 \\
(0,648-0,746)\end{array}$ & $\begin{array}{c}0,742 \\
(0,696-0,782)\end{array}$ & $\begin{array}{c}0,996 \\
(0,976-0,999)\end{array}$ & $\begin{array}{c}0,365 \\
(0,294-0,442)\end{array}$ \\
\hline Contrainmunoelectroforesis & 24,1 & $\begin{array}{c}0,989 \\
(0,943-0,998)\end{array}$ & $\begin{array}{c}0,790 \\
(0,740-0,832)\end{array}$ & $\begin{array}{c}0,838 \\
(0,780-0,871)\end{array}$ & $\begin{array}{c}0,996 \\
(0,976-0,999)\end{array}$ & $\begin{array}{c}0,599 \\
(0,521-0,672)\end{array}$ \\
\hline
\end{tabular}


CUADRO 3. Valores kappa $(k)$ de concordancia entre la técnica MB-Dot-ELISA y otras pruebas bacteriológicas e inmunológicas de diagnóstico de la infección por Neisseria meningitidis del grupo B

\begin{tabular}{lccr}
\hline \multirow{2}{*}{ Otras técnicas } & \multicolumn{3}{c}{ MB-Dot-ELISA } \\
\cline { 2 - 4 } & $k^{\mathrm{a}}$ & Significado de $k$ & $z$ \\
\hline Cultivo & 0,636 & Intermedio & 4,155 \\
Microscopia & 0,669 & Intermedio & 5,494 \\
Contrainmunoelectroforesis & 0,741 & intermedio & 7,101 \\
\hline
\end{tabular}

aTodos los valores de $k$ son estadísticamente significativos.

4,155 y 7,101. En cada caso se estimó que la concordancia entre los resultados de la prueba MB-Dot-ELISA y de los análisis bacteriológicos (microscopia o cultivo) o la CIE fue intermedia.

La reproducibilidad inter e intraensayo de la técnica MB-Dot-ELISA concordó totalmente con las de las pruebas iniciales. Por último, en lo que respecta a la estabilidad de las membranas de nitrocelulosa, se observó que, después de manchar las bandas de nitrocelulosa con las muestras de LCR, era posible guardarlas durante 30 días a $37^{\circ} \mathrm{C}$ o durante 6 meses a temperatura ambiente sin pérdida de reactividad.

\section{DISCUSIÓN}

En el diagnóstico de infecciones meningocócicas del grupo B, las prue- bas inmunológicas que permiten detectar antígenos bacterianos en los fluidos biológicos de los pacientes desempeñan un papel importante junto con las técnicas bacteriológicas, lo cual contribuye a prescribir la quimioterapia apropiada y a tomar otras medidas de control de epidemias.

En el presente estudio se estandarizó y evaluó la prueba MB-Dot-ELISA con antisuero policlonal para detectar el antígeno de $N$. meningitidis del grupo B en muestras de LCR de pacientes con meningitis.

Coll et al. (2), utilizando anticuerpos monoclonales, notificaron que el porcentaje de positividad de la prueba de inmunovaloración en mancha fue $67 \%$ al estudiar casos de meningitis meningocócica del grupo B confirmados con análisis bacteriológicos. En este estudio no se detectaron reaccio- nes positivas en las 13 muestras de LCR de casos de meningitis causados por otros agentes etiológicos, lo cual indica que la especificidad de la prueba fue $100 \%$.

Por el contrario, los análisis aquí notificados con la prueba MB-DotELISA y anticuerpos policlonales permitieron detectar casi todas $(99,1 \%)$ las infecciones del grupo B. Se observó alguna reactividad cruzada, pero la especificidad mejoró con las absorciones adicionales de antisuero de caballo con cada tipo de bacterias o una mezcla de bacterias con distinta reactividad cruzada, lo cual rindió una especificidad de 0,826 . Es preciso señalar que $83(84,7 \%)$ de las muestras de LCR que solamente fueron positivas en la microscopia para diplococos gramnegativos fueron positivas a N. meningitidis del grupo B con la prueba MB-DotELISA.

Se descubrió que la prueba MB-DotELISA, además de ser sumamente sensible, ofrece más ventajas que la CIE. En otras palabras, se redujo mucho la cantidad necesaria de antígeno y antisuero, no fue preciso utilizar equipo especial y fue fácil enviar para examen las tiras manchadas por correo habitual a laboratorios alejados. La principal conclusión es que esta prueba es sensible, barata y apropiada para las investigaciones que se realizan en los laboratorios de salud pública.

\section{REFERENCIAS}

1. Panachão MRI, Kallás EG, Barbosa HA, et al. Group B meningococcal disease in São Paulo, Brasil: an epidemiological and microbiological study. In: Evans JS, Yost SE, Maiden MCJ, Feavers IM, eds. Neisseria 94: proceedings of the Ninth International Pathogenic Neisseria Conference. Winchester, England: Guidhall; 1994:395.

2. Coll P, Borche L, Ausina V, Mirelis B, Prats G. Dot-immunobinding assay with a monoclonal antibody for detection of Group B meningococcal antigen. Eur J Clin Microbiol 1986;5: 44-46.

3. Ghanassia JP, Slim A, Bergogne-Berezin E, Modai J. Failure of diagnosing group B men- ingococcal meningitis by immunoelectrophoresis. Scand J Infect Dis 1977;9:313-314.

4. Alkmin MGA, Hoshino-Shimizu S, Landgraf IM, et al. Production and immunochemical characterization of Neisseria meningitidis group $\mathrm{B}$ antiserum for the diagnosis of purulent meningitis. Braz J Med Biol Res 1994;27: 16-34.

5. Balows A, Hausler WJ Jr, Herrmann KL, Isenberg HO, Shadomy HJ, eds. Manual of clinical microbiology. 5a ed. Washington, DC: American Society for Microbiology; 1991.

6. Brasil, Divisão Nacional de Laboratórios de Saúde Pública. Normas técnicas para o diagnóstico das meningites bacterianas. Brasília: Centro de Documentação do Ministério da Saúde; 1986:496. (Série A, normas e manuais técnicos, 32.)

7. Palhares M, Gelli DS, Almeida MCR, et al. Pesquisa de polissacarídeos de Neisseria meningitidis do grupo $\mathrm{C}$ no líquido cefalorraquidiano por imunoeletroforese cruzada em acetato de celulose. Rev Inst Adolfo Lutz 1984;33:85-89.

8. Gotschlich EC, Liu TY, Arnstein MS. Human immunity to the meningococcus: III, preparation and immunochemical properties of the group $\mathrm{A}$, group $\mathrm{B}$, and group $\mathrm{C}$ meningococcal polysaccharides. J Exp Med 1969;129: 1349-1365. 
9. Galen RJ, Gambino JR. Beyond normality: the predictive value and efficiency of medical diagnosis. New York: John Wiley; 1975:235.

10. Fleiss JL. Statistical methods for rates and proportions. New York: Wiley; 1981:217-225.

11. Maclure M, Willet WC. Misinterpretation and misuse of the kappa statistic. Am J Epidemiol 1987;126:161-169.
12. Feinstein AR. Clinical epidemiology. En: The architecture of clinical research. Philadelphia: Saunders; 1985:185-186.

13. Rothman KJ, Boice J. Epidemiologic analysis with programmable calculator. Boston: Epidemiology Resources; 1982.
Manuscrito recibido el 6 de febrero de 1995 y aceptado para publicación en versión revisada el 3 de noviembre de 1995.

ABSTRACT Infection with Neisseria meningitidis group B has been difficult to detect, partly because this bacterial group's polysaccharide is a weak immunogen. This article describes work carried out to test a new procedure (MB-Dot-ELISA) employing a high-titered horse antiserum for detection of $N$. meningitidis group $\mathrm{B}$ antigens. The study assayed cerebrospinal fluid samples from 585 subjects, 574 with suspected meningitis cases and 11 with neurologic disorders. The results of the assay indicated a sensitivity of 0.991 and a specificity of 0.826 . These results were superior to those obtained with latex agglutination and in substantial agreement with the results of counterimmunoelectrophoresis and bacteriologic methods. Overall, the MB-Dot-ELISA was found to be sensitive, inexpensive, and suitable for public health laboratory investigations.

ERRATA

Pellegrini Filho A, et al., "Producción de artículos científicos sobre salud en seis países de América Latina, 1973 a 1992"

(Rev Pan Am Salud Publica/Pan Am J Public Health 1997;1(1):23-34)

Se ruega a los lectores tomar nota de que las afiliaciones de los coautores Goldbaum y Silvi aparecen trastrocadas.

- Junto al nombre del segundo autor, Moisés Goldbaum, debería estar la llamada correspondiente a la nota 3, pues es miembro del Departamento de Medicina Preventiva y Social de la Facultad de Medicina de la Universidad de São Paulo, Brasil.

- John Silvi, en cambio, debería haberse identificado con una cuarta nota que indicara su afiliación con el Programa de Análisis de la Situación de Salud, División de Salud y Desarrollo, Organización Panamericana de la Salud.

La Redacción lamenta cualquier inconveniente que se haya presentado debido a este error. 